\title{
A Broadband Single-Stage Equivalent Circuit for Modeling LTCC Bandpass Filters
}

\author{
Yu-Shun Tsai and Tzyy-Sheng Horng, Senior Member, IEEE
}

\begin{abstract}
A single-stage equivalent circuit is proposed to model microwave bandpass filters (BPFs) fabricated in low-temperature co-fired ceramic (LTCC) technology up to several times its passband frequency. The equivalent circuit adopts a modified $T$ topology with the expandable multilayer resonators to achieve an extremely large bandwidth. It can be efficiently established from the measured $S$-parameters using direct extraction or rational approximation. As a result, the modeled $S$-parameters for a 2.45-GHz wideband local area network (WLAN) LTCC BPF show good agreement with the measured results over a wide frequency range up to $8.5 \mathrm{GHz}$. Such a broadband model can be used to accurately predict the suppression of harmonics and interferences in system simulation of the WLAN front-end modules.
\end{abstract}

Index Terms-Equivalent-circuit model, low-temperature co-fired ceramic (LTCC) bandpass filter (BPF), wideband local area network (WLAN) BPF.

\section{INTRODUCTION}

$\mathbf{I}$ $\mathrm{N}$ WIRELESS front-end applications, microwave bandpass filters (BPFs) are crucial components to suppressing the output harmonics in the transmitter and the input interferences in the receiver, as illustrated in Fig. 1. When connected with the power amplifiers or low-noise amplifiers, they possibly vary the load or source termination impedances at harmonic frequencies, which has an impact on linearity and efficiency of the amplifiers. However, the measured responses of $S$-parameters provided by the filter manufacturers have quite limited use in quantifying the suppression or termination effects. This is because accurate prediction of those effects generally requires a system-level nonlinear simulation. Broadband SPICE models should still be a must for microwave BPFs when used in the nonlinear simulation with active components.

Due to the complexity of all kinds of electromagnetic (EM) effects involved, the establishment of broadband models for microwave BPFs is still difficult and challenging. Previous methods include the physical models [1]-[3], EM simulation models [4]-[7], and model-based approaches [8]-[15]. The physical models can take the high-frequency losses, coupling, and parasitic effects into account, but require complete knowledge of every physical component inside a BPF. The EM simulation models can account for the EM phenomena clearly by partitioning the filter geometrical configuration and establishing

Manuscript received March 31, 2006; revised July 1, 2006. This work was supported in part by the Ministry of Education under the Program of Aim for the Top University Plan, Taiwan, R.O.C., and by the National Science Council, Taiwan, R.O.C., under Grant 94-2213-E-110-031.

The authors are with the RF and Microwave Laboratory, Department of Electrical Engineering, National Sun Yat-Sen University, Kaohsiung 804, Taiwan, R.O.C. (e-mail: d9031821@student.nsysu.edu.tw; jason@ee.nsysu.edu.tw).

Digital Object Identifier 10.1109/TMTT.2006.883603

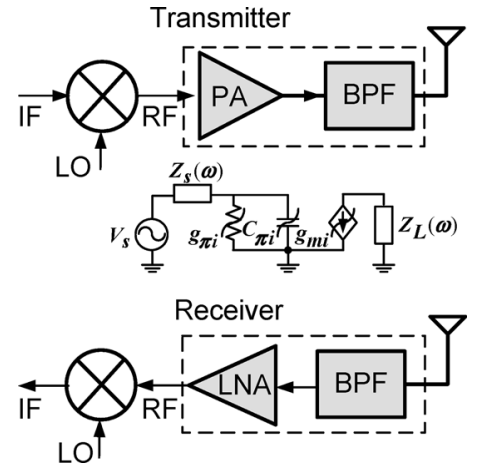

Fig. 1. Microwave BPFs causing the termination effects on the active components in the transmitter and receiver of a wireless front-end module.

the partial-element equivalent circuits (PEECs) based on the EM simulation results. However, EM simulation usually takes a long computation time. Besides, it is sometimes difficult to find a proper partition for accurate PEEC extraction without the help of optimization techniques. The model-based approaches can synthesize a real filter response and, thus, are quite helpful in diagnosing and tuning a BPF. However, they usually require the intensive optimization schemes to find the equivalent-circuit parameters [11]-[13]. In addition, the solutions may not be unique. Although in [10] an extraction procedure using the closed-form recursive formulas was demonstrated to find the equivalent-circuit parameters, the modeled filters were limited to some symmetric and known types. To the best of the authors' knowledge, the equivalent-circuit models for microwave BPFs reported to date in the literature were not yet able to cover a broad frequency range from direct current up to two or more integer times the passband center frequency.

In our previous research [16]-[18] for studying and modeling the passive components embedded in a multilayer low-temperature co-fired ceramic (LTCC) substrate, the modified T-equivalent circuit was first found suitable for broadband modeling of spiral inductors of arbitrary kind. Recently we explored the applications of such an equivalent-circuit topology to the LTCC BPFs, and the preliminary modeled results shown in [19] were quite encouraging. In this paper, we aim to provide an evolutionary insight into the broadband characteristics of modified T-equivalent circuit, and compare the accuracy and efficiency between two different approaches, i.e., direct extraction and rational approximation, in establishing the modified $\mathrm{T}$-equivalent circuit. It is emphasized that the proposed modified T-equivalent circuit is only a single-stage model, but can be easily expanded to achieve a bandwidth as large as a distributed circuit model. 


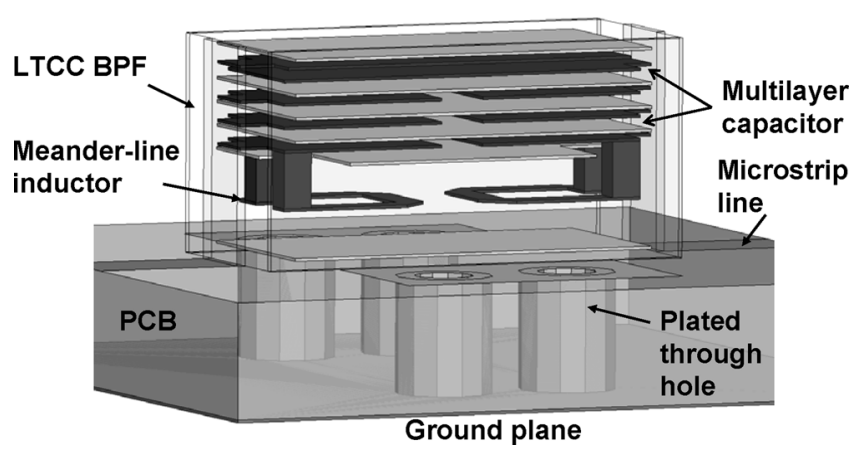

Fig. 2. Design configuration of the LTCC BPF.

\section{EVOLUTION OF BPF PROTOTYPE}

The currently available LTCC technology can implement high-performance miniature BPFs quite successfully because of the capability to realize large-value capacitors in a large number of thin substrate layers. To fully employ this capability, most of the LTCC BPF designs adopt a capacitive coupling of parallel resonators as a lumped-element prototype [20], [21], which is mainly composed of the large-value multilayer capacitors in conjunction with the small-value meander-line inductors, as illustrated in Fig. 2. The BPF configuration shown in Fig. 2 uses the capacitive coupling to link two parallel resonators. It has a small size advantage due to the geometrical simplicity. However, it is based on a second-order BPF prototype that has an inherent lower rolloff in comparison with a higher order design [22], [23]. To improve this drawback, a small ground inductance by means of a proper design of the LTCC ground electrodes and the plated through holes to printed circuit board (PCB) ground can be applied to create a transmission zero in both the lower and higher stopbands for enhancing the rolloff rates.

Fig. 3(a) shows a П-topology prototype with a series inductive feedback to account for the LTCC BPF configuration shown in Fig. 2. As shown in Fig. 3(b), this prototype can synthesize a transmission response with two angular transmission-zero frequencies, i.e., $\omega_{z 1}$ and $\omega_{z 2}$, at both sides of passband. Another view of this prototype is in a T-topology with a parallel capacitive feedback, as shown in Fig. 3(c). Generally speaking, the equivalent circuits established based on the prototype shown in Fig. 3(a) or (c) for an LTCC BPF can hardly explain the spurious behavior in the measured $S$-parameter responses. This is because the multilayer capacitors in an LTCC BPF are usually of so large a value as to cause the obvious series and higher order resonance effects [18], [24] in the frequency range of interest. It might be thought that the equivalent circuits, as suggested in [24], for modeling the multilayer capacitors with series and higher order resonances can be included. However, this makes the extraction of the equivalent-circuit elements from the measured $S$-parameters become a formidable task.

This paper presents an alternative prototype based on the modified T topology shown in Fig. 3(d) as a foundation to establish the broadband equivalent circuits for LTCC BPFs. The new prototype has a unique feature to put all the capacitance

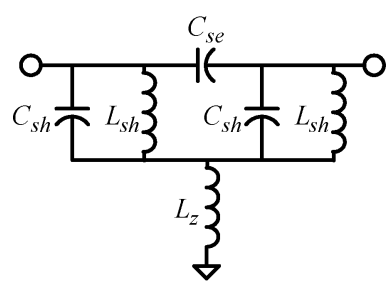

(a)

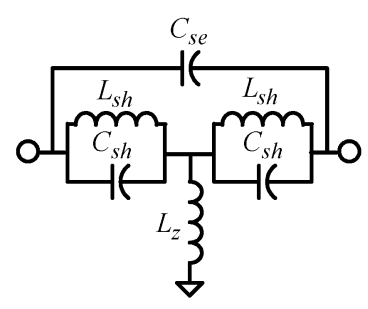

(c)

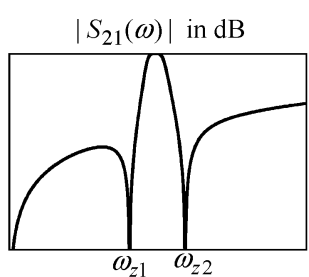

(b)

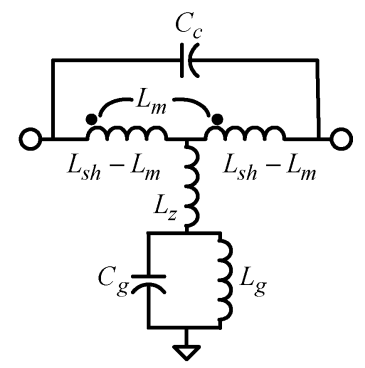

(d)
Fig. 3. (a) LTCC BPF prototype in $\Pi$ topology with a series inductive feedback. (b) Magnitude of $S_{21}$ in decibel generated from the LTCC BPF prototype. (c) LTCC BPF prototype in T topology with a parallel capacitive feedback. (d) LTCC BPF prototype in modified T topology.

elements $\left(C_{c}\right.$ and $\left.C_{g}\right)$ in the parallel and series feedback circuits. Included with the additional mutual and shunt inductance elements $\left(L_{m}\right.$ and $L_{g}$ ), the modified T-topology prototype shown in Fig. 3(d) can be equivalent to the prototype shown in Fig. 3(a) or (c) if the following relations hold:

$$
\begin{aligned}
L_{g} & =L_{m}=\frac{L_{s h}}{2} \\
C_{g} & =2 C_{s h} \\
C_{c} & =\frac{C_{s h}}{2}+C_{s e} .
\end{aligned}
$$

It is emphasized that such a modified T-topology prototype for modeling an LTCC BPF is easy to include the series and higher order resonances resulting from the multilayer capacitors by expanding the series and parallel feedback circuits as the multilayer resonators. In addition, direct extraction or rational approximation can be applied to find all the equivalent-circuit elements from the measured $S$-parameters. The detailed procedures are provided in Sections IV and V.

\section{Modified T-EQuivalent Circuit}

Fig. 4(a) shows a generalized modified T-equivalent network proposed for modeling LTCC BPFs. When compared to the well-known T-equivalent network, the modified T-equivalent network additionally includes the parallel-feedback admittance $\left(Y_{p}\right)$, series-feedback impedance $\left(Z_{g}\right)$, and mutual inductive impedance $\left(Z_{m}\right)$ to model the coupling and grounding effects. By modeling $Y_{p} / Z_{g}$ as a multilayer series/parallel resonator with a continuous increase of layer numbers, as illustrated in Fig. 4(b), the modified T-equivalent circuit can expand accordingly to an increase in the bandwidth in a way like a distributed circuit, but still remains a single-stage equivalent circuit. 


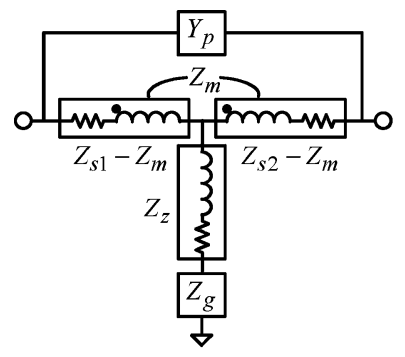

(a)

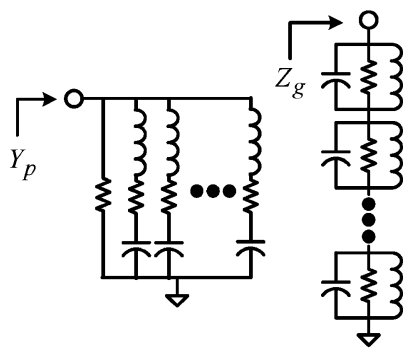

(b)
Fig. 4. (a) Proposed modified T-equivalent network. (b) Use of expandable multilayer resonators for modeling the high-frequency resonance effects.

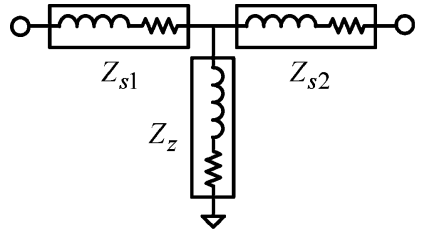

(a)

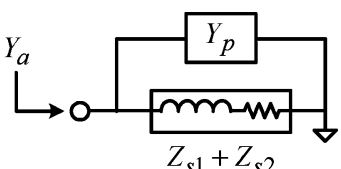

(b)

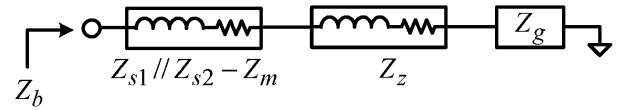

(c)

Fig. 5. (a) Reduced T-equivalent network at low frequency. (b) Decomposed one-port network with input admittance of $Y_{a}$ according to (10). (c) Decomposed one-port network with input impedance of $Z_{b}$ according to (11).

At low frequencies, the coupling and grounding effects are negligible, which means mathematically that $Y_{p} \rightarrow 0$ and $Z_{m} \rightarrow Z_{g}$ as $\omega \rightarrow 0$. With this condition, the modified T-equivalent network can reduce to a simple T-equivalent network composed of two series impedance $\left(Z_{s 1}, Z_{s 2}\right)$ and one shunt impedance $\left(Z_{z}\right)$ only, as shown in Fig. 5(a). When all types of coupling and grounding effects need to be considered at high frequencies, the modified T-equivalent network, as shown in Fig. 4(a), can be generally used. After derivation, its $Z$ and $Y$ network parameters are expressed as

$$
\begin{aligned}
Z_{11} & =\frac{Z_{s 1}+Y_{p} Z_{s 1} Z_{s 2}}{1+Y_{p}\left(Z_{s 1}+Z_{s 2}\right)}+\left(Z_{z}+Z_{g}-Z_{m}\right) \\
Z_{22} & =\frac{Z_{s 2}+Y_{p} Z_{s 1} Z_{s 2}}{1+Y_{p}\left(Z_{s 1}+Z_{s 2}\right)}+\left(Z_{z}+Z_{g}-Z_{m}\right) \\
Z_{12} & =Z_{21}=\frac{Y_{p} Z_{s 1} Z_{s 2}}{1+Y_{p}\left(Z_{s 1}+Z_{s 2}\right)}+\left(Z_{z}+Z_{g}-Z_{m}\right) \\
Y_{11} & =\frac{Z_{s 2}+Z_{z}+Z_{g}-Z_{m}}{Z_{s 1} Z_{s 2}+\left(Z_{s 1}+Z_{s 2}\right)\left(Z_{z}+Z_{g}-Z_{m}\right)}+Y_{p} \\
Y_{22} & =\frac{Z_{s 1}+Z_{z}+Z_{g}-Z_{m}}{Z_{s 1} Z_{s 2}+\left(Z_{s 1}+Z_{s 2}\right)\left(Z_{z}+Z_{g}-Z_{m}\right)}+Y_{p} \\
Y_{12} & =Y_{21}=\frac{-\left(Z_{z}+Z_{g}-Z_{m}\right)}{Z_{s 1} Z_{s 2}+\left(Z_{s 1}+Z_{s 2}\right)\left(Z_{z}+Z_{g}-Z_{m}\right)}-Y_{p} .
\end{aligned}
$$

From (4)-(9), we find that the two-port modified T-equivalent network can be decomposed into two one-port networks with input impedance or admittance defined ass

$$
\begin{aligned}
& Y_{a}=\left(Z_{11}+Z_{22}-2 Z_{12}\right)^{-1}=\frac{1}{Z_{s 1}+Z_{s 2}}+Y_{p} \\
& Z_{b}=\left(Y_{11}+Y_{22}+2 Y_{12}\right)^{-1}=\frac{Z_{s 1} Z_{s 2}}{Z_{s 1}+Z_{s 2}}-Z_{m}+Z_{z}+Z_{g}
\end{aligned}
$$

From (10), one can know that $Y_{a}$ is equal to the resulting admittance for the total of the main series impedance $\left(Z_{s 1}+Z_{s 2}\right)$ in parallel connection with the parallel-feedback admittance $\left(Y_{p}\right)$, as depicted in Fig. 5(b). From (11), $Z_{b}$ can be looked upon as the impedance of a series connection of the shunt impedance $\left(Z_{z}\right)$, series-feedback impedance $\left(Z_{g}\right)$, and an impedance equal to the parallel connection of two series impedances $\left(Z_{s 1} / / Z_{s 2}\right)$ subtracting the mutual inductive impedance $\left(Z_{m}\right)$, as depicted in Fig. 5(c). It is noted that, according to (10) and (11), $Y_{a}$ and $Z_{b}$ can be practically obtained from $Z$ and $Y$ network parameters, respectively, converted from the measured or EM-simulated $S$-parameters.

As a matter of fact, the proposed modified T-network topology can be used to establish the mathematically equivalent circuit of any two-port reciprocal component. However, to consider the physical aspects, the actual representation of equivalent circuits using a modified T-network topology in this paper includes the low-frequency extracted parameters and the multilayer resonators to account for the prototype configuration and the high-frequency resonance effects, respectively, for specific use on the LTCC BPFs.

\section{DiRECT EXTRACTION}

For our study case of a $2.45-\mathrm{GHz}$ LTCC BPF, it is quite straightforward to extract the equivalent series $L R$ circuits for $Z_{s 1}, Z_{s 2}$, and $Z_{z}$ in Fig. 5(a) from the $Z$ network parameters at low frequencies

$$
\begin{aligned}
Z_{s 1} & =R_{s 1}+j \omega L_{s 1} \approx Z_{11}-Z_{12}, \quad \text { for low } \omega \\
Z_{s 2} & =R_{s 2}+j \omega L_{s 2} \approx Z_{22}-Z_{12}, \quad \text { for low } \omega \\
Z_{z} & =R_{z}+j \omega L_{z} \approx Z_{12}, \quad \text { for low } \omega .
\end{aligned}
$$

Extraction of the other equivalent circuits for $Z_{m}, Y_{p}$, and $Z_{g}$ can rely on input admittance/impedance of the decomposed networks, $Y_{a}$ and $Z_{b}$, with the suggested equivalent circuit shown in Fig. 6(a) and (b), respectively. $Z_{m}$ represents the impedance of the mutual inductance $\left(L_{m}\right)$ that appears in the BPF prototype configuration of Fig. 3(d). $Y_{p}$ and $Z_{g}$ are modeled as the expandable multilayer resonators to account for the higher order resonance effects due to coupling and grounding, respectively.

The $Y_{p}$ resonant circuit elements can be extracted from the frequency response of $Y_{a}$. To determine the reactive elements in the $Y_{p}$ resonant circuit that is composed of a number of $j$ series resonators, we need to identify a number of $j$ pairs of angular parallel and series resonant frequencies, i.e., $\omega_{c p i}$ and $\omega_{c s i}$, and $i=1,2,3, \ldots, j$, in the imaginary response of $Y_{a}$ within the measurement frequency range, as shown in Fig. 7. 


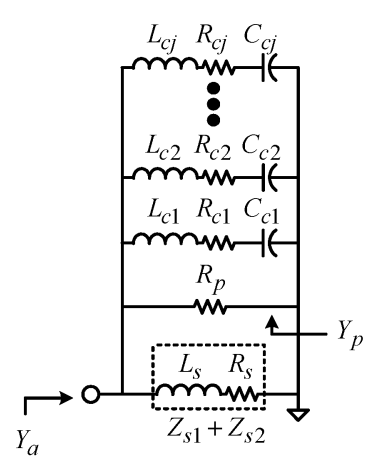

(a)

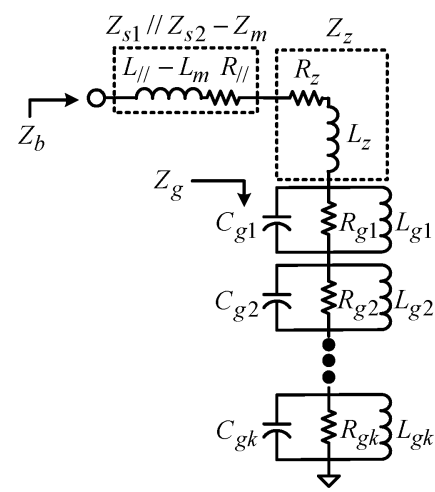

(b)
Fig. 6. Suggested equivalent circuits for modeling the decomposed one-port networks. (a) $Y_{a}$ network. (b) $Z_{b}$ network.

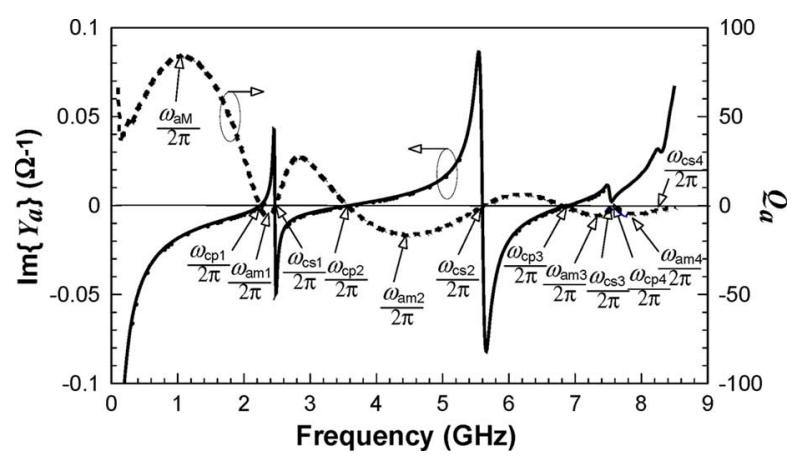

Fig. 7. Specific frequencies used for extracting the $Y_{p}$ resonant circuit elements in the direct-extraction procedure.

The formulated equation for reactive elements at these resonant frequencies is written as

$$
\frac{-1}{\omega_{c p i}^{2} L_{s}}+\sum_{i^{\prime}=1}^{j} \frac{C_{c i^{\prime}}}{1-\left(\omega_{c p i} / \omega_{c s i^{\prime}}\right)^{2}}=0, \quad i=1,2,3, \ldots, j
$$

where $L_{s}=L_{s 1}+L_{s 2}$, which is the inductance extracted from the imaginary part of $Z_{s 1}+Z_{s 2}$ at low frequencies. Solving (15) yields

$$
\begin{aligned}
& C_{c i}= \frac{(-1)^{i}}{L_{s} \omega_{c s i}^{2}} \prod_{n, m=1}^{j} \frac{\omega_{c s n}^{2}\left(\omega_{c p m}^{2}-\omega_{c s i}^{2}\right)}{\omega_{c p m}^{2}\left(\omega_{c s i}^{2}-\omega_{c s n}^{2}\right)}, \\
& \quad \text { for } n \neq i \text { and } i \leq j \\
& L_{c i}=\frac{1}{C_{c i} \omega_{c s i}^{2}} .
\end{aligned}
$$

In this approach, the resistances in the $Y_{p}$ resonant circuit are extracted from $Y_{a}$ 's $Q$-factor $\left(Q_{a}\right)$ response, as shown in Fig. 7 rather than $Y_{a}$ 's real response. The reasons are because the former is more closely related to the frequency dependence of insertion loss in an LTCC BPF and also behaves more smoothly than the latter. The definition of $Q_{a}$ is given as

$$
Q_{a}=-\frac{\operatorname{Im}\left\{Y_{a}\right\}}{\operatorname{Re}\left\{Y_{a}\right\}}
$$

The parallel resistance $\left(R_{p}\right)$ can be determined from the first peak $Q_{a}$ factor $\left(Q_{a M}\right)$ and its corresponding angular frequency $\left(\omega_{a M}\right)$ using the following approximation of $Y_{a}$ under the lowfrequency condition:

$$
Y_{a}\left(\omega_{a M}\right) \approx \frac{1}{R_{p}}+\frac{1}{R_{s}+j \omega_{a M} L_{s}}+j \omega_{a M} C_{s}
$$

where

$$
C_{s}=\sum_{i=1}^{j} C_{c i} .
$$

In (19), $R_{s}=R_{s 1}+R_{s 2}$, which is the resistance in series with $L_{s}$ and can be extracted from the real part of $Z_{s 1}+Z_{s 2}$ at low frequencies. Substituting (19) into (18) and setting $Q_{a}$ equal to $Q_{a M}$, one can solve for $R_{p}$ as

$$
R_{p} \approx \frac{Q_{a M}\left(R_{s}^{2}+\omega_{a M}^{2} L_{s}^{2}\right)}{\omega_{a M}\left[L_{s}-C_{s}\left(R_{s}^{2}+\omega_{a M}^{2} L_{s}^{2}\right)\right]-Q_{a M} R_{s}} .
$$

The other resistances, i.e., $R_{c i}, i=1,2,3, \ldots, j$, are determined from identifying a number of $j$ local minima, i.e., $Q_{a m i}$ at $\omega_{\text {ami }}, i=1,2,3, \ldots, j$, as depicted in Fig. 7. The formulation can be described as

$$
Q_{a m i}=-\frac{\operatorname{Im}\left\{Y_{a}\left(\omega_{a m i}\right)\right\}}{\operatorname{Re}\left\{Y_{a}\left(\omega_{a m i}\right)\right\}}, \quad i=1,2,3, \ldots, j
$$

where

$$
\begin{aligned}
Y_{a}\left(\omega_{a m i}\right)= & \frac{1}{R_{p}}+\frac{1}{R_{s}+j \omega_{a m i} L_{s}} \\
& +\sum_{i^{\prime}=1}^{j} \frac{j \omega_{a m i} C_{c i^{\prime}}}{1+j \omega_{a m i} C_{c i^{\prime}} R_{c i^{\prime}}-\left(\omega_{a m i} / \omega_{c s i^{\prime}}\right)^{2}} .
\end{aligned}
$$

After substituting (23) into (22), one can solve for $R_{c i}, i=$ $1,2,3, \ldots, j$ from the following set of equations:

$$
\begin{aligned}
\frac{Q_{a m i} R_{s}-\omega_{a m i} L_{s}}{R_{s}^{2}+\omega_{a m i}^{2} L_{s}^{2}}+\frac{Q_{a m i}}{R_{p}} & +\sum_{i^{\prime}=1}^{j} \frac{Q_{a m i} R_{c i^{\prime}}-U_{i i^{\prime}}}{R_{c i^{\prime}}^{2}+U_{i i^{\prime}}^{2}} \\
& =0, \quad i=1,2,3, \ldots, j
\end{aligned}
$$

where

$$
U_{i i^{\prime}}=\frac{\omega_{a m i}^{2}-\omega_{c s i^{\prime}}^{2}}{\omega_{a m i} C_{c i^{\prime}} \omega_{c s i^{\prime}}^{2}} .
$$

A side advantage using the $Q_{a}$ response deserves to be mentioned that the frequencies at which $Q_{a}$ becomes zero correspond to the parallel or series resonant frequencies of $Y_{a}$. This provides a fast way of getting those resonant frequencies data for extracting the reactive elements in the $Y_{p}$ resonant circuit.

In a similar fashion, by identifying the parallel and series angular resonant frequencies, i.e., $\omega_{g p i}$ and $\omega_{g s i}, i=1,2,3, \ldots, k$ in the imaginary response of $Z_{b}$, as shown in Fig. 8, we can find $L_{m}$ and all the reactive elements in the $Z_{g}$ resonant circuit shown in Fig. 6(b) from the following formulation:

$$
\begin{aligned}
\omega_{g s i}\left(L_{/ /}-L_{m}+L_{z}\right)+\sum_{i^{\prime}=1}^{k} & \frac{\omega_{g s i} L_{g i^{\prime}}}{1-\left(\omega_{g s i} / \omega_{g p i^{\prime}}\right)^{2}} \\
& =0, \quad i=1,2,3, \ldots, k
\end{aligned}
$$




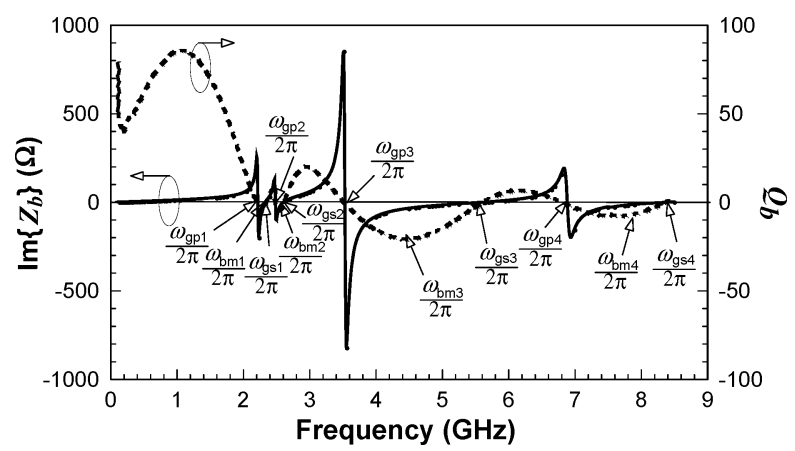

Fig. 8. Specific frequencies used for extracting the $Z_{g}$ resonant circuit elements in the direct-extraction procedure.

where $L_{/ /}$and $L_{z}$ are the inductances extracted from the imaginary part of $Z_{s 1} / / Z_{s 2}$ and $Z_{z}$, respectively, at low frequencies. Note that $Z_{m} \rightarrow Z_{g}$ as $\omega \rightarrow 0$, which implies

$$
L_{m}=\sum_{i=1}^{k} L_{g i}
$$

Solving (26) yields

$$
\begin{aligned}
L_{g i} & =\frac{-\left|L_{/ /}-L_{m}+L_{z}\right|}{\omega_{g p i}^{2}} \prod_{n, m=1}^{k} \frac{\left(\omega_{g p i}^{2}-\omega_{g s m}^{2}\right)}{\left(\omega_{g p i}^{2}-\omega_{g p n}^{2}\right)}, \\
\text { for } n \neq i \text { and } i \leq k & \frac{1}{L_{g i} \omega_{g p i}^{2}} .
\end{aligned}
$$

As for the resistances, i.e., $R_{g i}, i=1,2,3, \ldots, k$, in the $Z_{g}$ resonant circuit, they are determined from $Z_{b}$ 's $Q$ factor $\left(Q_{b}\right)$ defined as

$$
Q_{b}=\frac{\operatorname{Im}\left\{Z_{b}\right\}}{\operatorname{Re}\left\{Z_{b}\right\}} .
$$

By identifying a number of $k$ local minima, $Q_{b m i}$ at $\omega_{b m i}, i=$ $1,2,3, \ldots, k$ in the $Q_{b}$ response, as shown in Fig. 8, we can express $Q_{b m i}$ as

$$
Q_{b m i}=\frac{\operatorname{Im}\left\{Z_{b}\left(\omega_{b m i}\right)\right\}}{\operatorname{Re}\left\{Z_{b}\left(\omega_{b m i}\right)\right\}}, \quad i=1,2,3, \ldots, k
$$

where

$$
\begin{aligned}
Z_{b}\left(\omega_{b m i}\right)= & R_{/ /}+R_{z}+j \omega_{b m i}\left(L_{/ /}-L_{m}+L_{z}\right) \\
& +\sum_{i^{\prime}=1}^{k} \frac{j \omega_{b m i} L_{g i^{\prime}}}{1+j \omega_{b m i} L_{g i^{\prime}} / R_{g i^{\prime}}-\left(\omega_{b m i} / \omega_{g p i^{\prime}}\right)^{2}} .
\end{aligned}
$$

In (32), $R_{/ /}$and $R_{z}$ are the resistances extracted from the real part of $Z_{s 1} / / Z_{s 2}$ and $Z_{z}$, respectively, at low frequencies. Substituting (32) into (31), one can solve for $R_{g i}, i=1,2,3, \ldots, k$ from the following set of equations:

$$
\begin{aligned}
& Q_{b m i}\left(R_{/ /}+R_{z}\right)-\omega_{b m i}\left(L_{/ /}-L_{m}+L_{z}\right) \\
& +\sum_{i^{\prime}=1}^{k} \frac{Q_{b m i} / R_{g i^{\prime}}+V_{i i^{\prime}}}{1 / R_{g i^{\prime}}^{2}+V_{i i^{\prime}}^{2}}=0, \quad i=1,2,3, \ldots, k
\end{aligned}
$$

where

$$
V_{i i^{\prime}}=\frac{\omega_{b m i}^{2}-\omega_{g p i^{\prime}}^{2}}{\omega_{b m i} L_{g i^{\prime}} \omega_{g p i^{\prime}}^{2}}
$$

\section{RATIONAL APPROXIMATION}

Inspired from the equivalent-circuit configurations shown in Fig. 6(a) and (b), we consider the rational approximation of $Y_{a}(s)$ and $Z_{b}(s)$ in the following forms:

$$
\begin{aligned}
& Y_{a}(s) \approx c+\frac{r_{0}}{s-p_{0}}+\sum_{i=1}^{j} F_{i}(s) \\
& Z_{b}(s) \approx d+s e+\sum_{i=1}^{k} F_{i}(s)
\end{aligned}
$$

where

$$
F_{i}(s)=\frac{r_{i}}{s-p_{i}}+\frac{r_{i}^{*}}{s-p_{i}^{*}} .
$$

Note that in (35)-(37), $c, d$, and $e$ are real constants, $r_{0}$ and $p_{0}$ denote the real residue and the real pole, respectively, and $\left(r_{i}, r_{i}^{*}\right)$ and $\left(p_{i}, p_{i}^{*}\right)$ for $i \geq 1$ are pairs of complex and conjugate residues and poles, respectively. All these constants, residues, and poles in (35)-(37) are determined based on a well-known vector-fitting procedure [25]-[28]. Under low-loss condition, $F_{i}(s)$ standing for the transfer function of a complex pole pair can be approximated as

$$
\begin{aligned}
F_{i}(s) & =\frac{s\left(r_{i}+r_{i}^{*}\right)-\left(r_{i} p_{i}^{*}+r_{i}^{*} p_{i}\right)}{s^{2}-s\left(p_{i}+p_{i}^{*}\right)+p_{i} p_{i}^{*}} \\
& \approx \frac{s\left(r_{i}+r_{i}^{*}\right)}{s^{2}-s\left(p_{i}+p_{i}^{*}\right)+p_{i} p_{i}^{*}} .
\end{aligned}
$$

On the other hand, $Y_{a}(s)$ and $Z_{b}(s)$ according to the equivalent circuits shown in Fig. 6(a) and (b) can be derived as

$$
\begin{aligned}
Y_{a}(s)= & \frac{1}{R_{p}}+\frac{1}{R_{s}+s L_{s}}+\sum_{i=1}^{j} \frac{s \frac{1}{L_{c i}}}{s^{2}+s \frac{R_{c i}}{L_{c i}}+\frac{1}{L_{c i} C_{c i}}} \\
Z_{b}(s)= & R_{/ /}+R_{z}+s\left(L_{/ /}+L_{z}-L_{m}\right) \\
& +\sum_{i=1}^{k} \frac{s \frac{1}{C_{g i}}}{s^{2}+s \frac{1}{R_{g i} C_{g i}}+\frac{1}{L_{g i} C_{g i}}} .
\end{aligned}
$$

By comparing (39) and (40) with (35) and (36), the relations between the equivalent-circuit elements and the vector-fitting parameters are found as

$$
\begin{aligned}
Z_{s}(s) & =R_{s}+s L_{s}=\frac{s-p_{0}}{r_{0}} \\
Z_{x}(s) & =R_{/ /}+R_{z}+s\left(L_{/ /}+L_{z}-L_{m}\right) \\
& =d+s e \\
R_{p} & =\frac{1}{c}
\end{aligned}
$$




$$
\begin{aligned}
L_{c i}, C_{g i} & =\frac{1}{r_{i}+r_{i}^{*}} \\
C_{c i}, L_{g i} & =\frac{r_{i}+r_{i}^{*}}{p_{i} p_{i}^{*}} \\
R_{c i}, \frac{1}{R_{g i}} & =-\frac{p_{i}+p_{i}^{*}}{r_{i}+r_{i}^{*}} .
\end{aligned}
$$

Thus far, we still need to determine $Z_{s 1}(s), Z_{s 2}(s)$, and $Z_{z}(s)$ before combining the two one-port equivalent circuits for $Y_{a}(s)$ and $Z_{b}(s)$ into the wanted two-port modified T-equivalent circuit for the LTCC BPF. By referring to Fig. 6(a) and (b), we know that

$$
\begin{aligned}
& Z_{s}(s)=Z_{s 1}(s)+Z_{s 2}(s) \\
& Z_{x}(s)=\frac{Z_{s 1}(s) Z_{s 2}(s)}{Z_{s 1}(s)+Z_{s 2}(s)}+Z_{z}(s)-s L_{m}
\end{aligned}
$$

where $L_{m}$ is a known element from (27). Another condition can be obtained by estimating the ratio of $Z_{s 1}(s)$ to $Z_{s 2}(s)$ in terms of the $Z$ network parameters at low frequencies, which is given as

$$
\gamma=\frac{Z_{s 1}}{Z_{s 2}} \approx \frac{Z_{11}-Z_{12}}{Z_{22}-Z_{12}}, \quad \text { for low } \omega
$$

From (47)-(49), $Z_{s 1}(s), Z_{s 2}(s)$, and $Z_{z}(s)$ can be finally determined as

$$
\begin{aligned}
& Z_{s 1}(s) \approx Z_{s}(s) \frac{\gamma}{1+\gamma} \\
& Z_{s 2}(s) \approx Z_{s}(s) \frac{1}{1+\gamma} \\
& Z_{z}(s) \approx Z_{x}(s)-Z_{s}(s) \frac{\gamma}{(1+\gamma)^{2}}+s L_{m} .
\end{aligned}
$$

\section{Modeled Results AND Discussion}

An LTCC BPF for 2.45-GHz wideband local area network (WLAN) applications was implemented according to the configuration shown in Fig. 2 as our modeling example. The BPF was designed to have an insertion loss less than $2.5 \mathrm{~dB}$ in the passband frequency range from 2.4 to $2.5 \mathrm{GHz}$, and an attenuation more than $25 \mathrm{~dB}$ at the second and third harmonic frequencies. To create a transmission zero at both sides of the passband for enhancing the rolloff rate, a small ground inductance was provided, resulting from the plated through holes in the PCB that serves as a mounting substrate. The $S$-parameter measurement for this LTCC BPF was taken up to $8.5 \mathrm{GHz}$ to cover higher than the third harmonic frequency. The measured results met our design goals and also proved our prediction to have the two transmission zeros at approximately 2 and $3 \mathrm{GHz}$.

Converted from the measured $S$-parameters, the two crucial parameters, i.e., $Y_{a}$ and $Z_{b}$, for establishing the modified T-equivalent circuit were processed through the procedure of direct extraction and rational approximation described in Sections IV and V, respectively, to determine and compare

\begin{tabular}{|c|c|c|c|c|}
\hline \multirow{9}{*}{$Y_{a}$} & $\omega_{c s 1}$ & $15.5068 \mathrm{e} 9$ & $\omega_{c p 1}$ & $14.0790 \mathrm{e} 9$ \\
\hline & $\omega_{c s 2}$ & $35.2845 \mathrm{e} 9$ & $\omega_{c p 2}$ & $22.4619 \mathrm{e} 9$ \\
\hline & $\omega_{c s 3}$ & $47.5932 \mathrm{e} 9$ & $\omega_{c p 3}$ & $42.7578 \mathrm{e} 9$ \\
\hline & $\omega_{c s 4}$ & $53.5441 \mathrm{e} 9$ & $\omega_{c p 4}$ & $48.0094 \mathrm{e} 9$ \\
\hline & $\omega_{a M}$ & $7.97965 \mathrm{e} 9$ & $Q_{a M}$ & 95.42 \\
\hline & $\omega_{a m 1}$ & $14.8283 \mathrm{e} 9$ & $Q_{a m 1}$ & -4.55 \\
\hline & $\omega_{a m 2}$ & $27.8345 \mathrm{e} 9$ & $Q_{a m 2}$ & -16.74 \\
\hline & $\omega_{a m 3}$ & $45.8044 \mathrm{e} 9$ & $Q_{a m 3}$ & -5.29 \\
\hline & $\omega_{a m 4}$ & $49.6372 \mathrm{e} 9$ & $Q_{a m 4}$ & -6.69 \\
\hline \multirow{8}{*}{$Z_{b}$} & $\omega_{g s 1}$ & $14.6423 \mathrm{e} 9$ & $\omega_{g p 1}$ & $13.9211 \mathrm{e} 9$ \\
\hline & $\omega_{g s 2}$ & $16.3509 \mathrm{e} 9$ & $\omega_{g p 2}$ & $15.5848 \mathrm{e} 9$ \\
\hline & $\omega_{g s 3}$ & $35.1511 \mathrm{e} 9$ & $\omega_{g p 3}$ & $22.1757 \mathrm{e} 9$ \\
\hline & $\omega_{g s 4}$ & $52.5083 \mathrm{e} 9$ & $\omega_{g p 4}$ & $42.7552 \mathrm{e} 9$ \\
\hline & $\omega_{b m 1}$ & $14.3257 \mathrm{e} 9$ & $Q_{b m 1}$ & -2.58 \\
\hline & $\omega_{b m 2}$ & $15.9593 \mathrm{e} 9$ & $Q_{b m 2}$ & -2.48 \\
\hline & $\omega_{b m 3}$ & $27.9602 \mathrm{e} 9$ & $Q_{b m 3}$ & -18.19 \\
\hline & $\omega_{b m 4}$ & $47.3124 \mathrm{e} 9$ & $Q_{b m 4}$ & -3.55 \\
\hline
\end{tabular}
the equivalent-circuit elements. To have a fair comparison, the above two different modeling approaches were conducted on purpose to construct the equivalent circuits of identical configuration with the same number of elements. Tables I and II show the necessary extracted data in both procedures for evaluating
TABLE I

PARAMETER VALUES USED IN THE DiRECT-EXTRACTION PROCEDURE FOR EVALUATING THE EQUIVALENT-CIRCUIT ELEMENTS

TABLE II

PARAMETER VALUES USED IN THE RATIONAL-APPROXIMATION PROCEDURE FOR EVALUATING THE EQUIVALENT-CIRCUIT ELEMENTS

\begin{tabular}{|c|c|c|c|c|}
\hline \multirow{5}{*}{$Y_{a}$} & $p_{1}$ & $-1.5093 \mathrm{e} 8 \pm j 1.5504 \mathrm{e} 10$ & $r_{1}$ & $7.9064 \mathrm{e} 6 \pm j 1.5587 \mathrm{e} 4$ \\
\cline { 2 - 5 } & $p_{2}$ & $-4.1695 \mathrm{e} 8 \pm j 3.5202 \mathrm{e} 10$ & $r_{2}$ & $6.5807 \mathrm{e} 7 \pm j 6.2169 \mathrm{e} 5$ \\
\cline { 2 - 5 } & $p_{3}$ & $-6.6280 \mathrm{e} 8 \pm j 4.7153 \mathrm{e} 10$ & $r_{3}$ & $5.7937 \mathrm{e} 6 \pm j 1.0096 \mathrm{e} 5$ \\
\cline { 2 - 6 } & $p_{4}$ & $-8.4316 \mathrm{e} 8 \pm j 5.5338 \mathrm{e} 10$ & $r_{4}$ & $1.5605 \mathrm{e} 8 \pm j 2.1619 \mathrm{e} 6$ \\
\hline \multirow{5}{*}{$z_{b}$} & $-5.1288 \mathrm{e} 7$ & $r_{0}$ & $1.2388 \mathrm{e} 8$ \\
\hline & $p_{0}$ & $1.7268 \mathrm{e}-5$ & & \\
\hline & $p_{1}$ & $-1.6556 \mathrm{e} 8 \pm j 1.3920 \mathrm{e} 10$ & $r_{1}$ & $3.7285 \mathrm{e} 10 \pm j 4.4348 \mathrm{e} 10$ \\
\hline & $p_{2}$ & $-6.4618 \mathrm{e} 7 \pm j 1.5590 \mathrm{e} 10$ & $r_{2}$ & $1.9747 \mathrm{e} 10 \pm j 8.1850 \mathrm{e} 7$ \\
\hline & $p_{4}$ & $-1.6046 \mathrm{e} 8 \pm j 2.2173 \mathrm{e} 10$ & $r_{3}$ & $2.3299 \mathrm{e} 11 \pm j 1.6861 \mathrm{e} 9$ \\
\cline { 2 - 6 } & $d$ & $0.0799 \mathrm{e} 8 \pm j 4.3258 \mathrm{e} 10$ & $r_{4}$ & $1.1912 \mathrm{e} 11 \pm j 8.4821 \mathrm{e} 8$ \\
\hline
\end{tabular}

the equivalent-circuit elements. It is noted that the direct-extraction approach utilized the $Q$-factor responses of $Y_{a}$ and $Z_{b}$ to find the resonant frequencies, as well as the local maxima and minima points listed in Table I and substituted them into (16), 


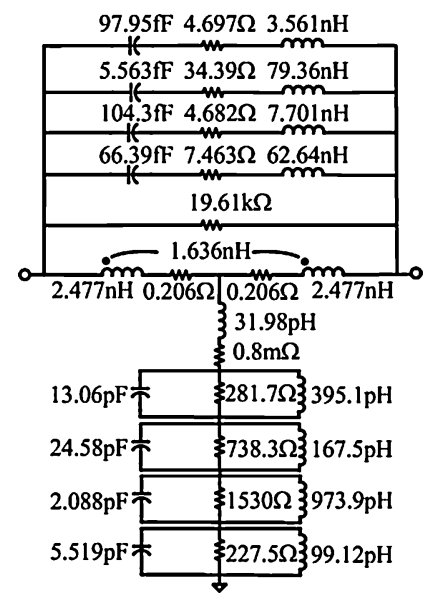

(a)

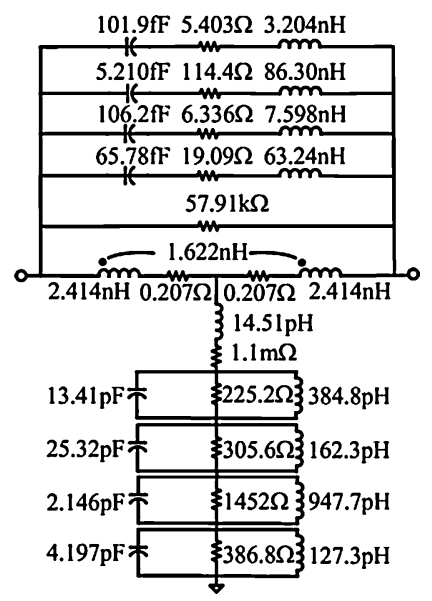

(b)
Fig. 9. Established modified T-equivalent circuits for the 2.45-GHz LTCC BPF. (a) Direct extraction method. (b) Rational approximation method.

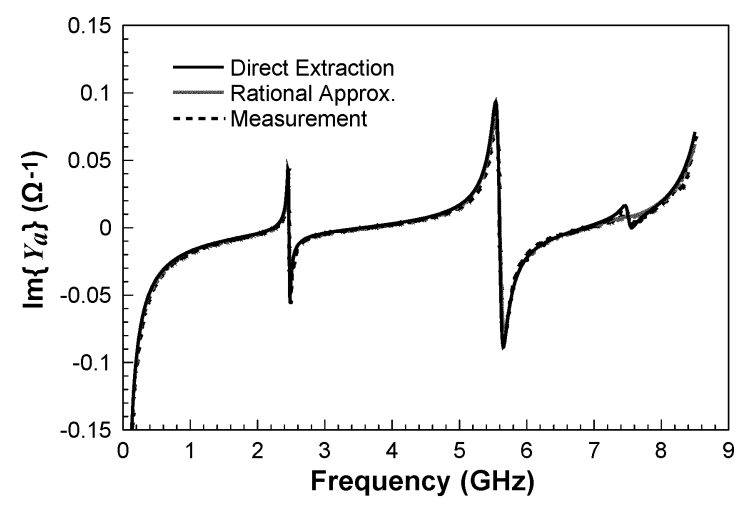

(a)

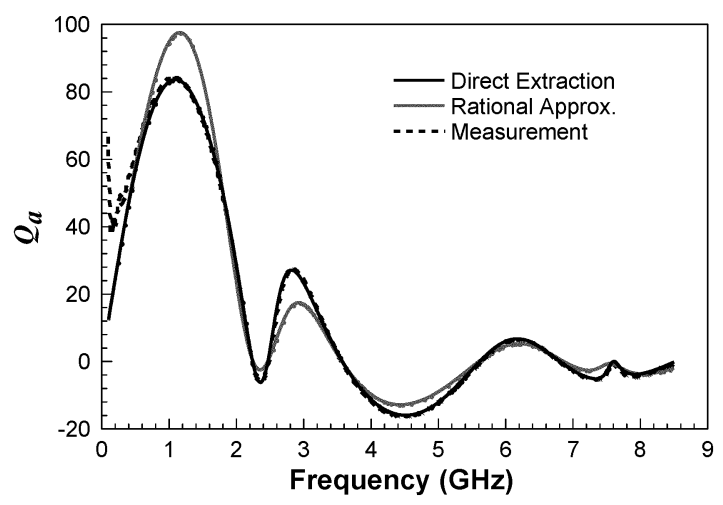

(b)

Fig. 10. Comparisons of the modeled results with the measurements for the one-port network with input admittance of $Y_{a}$. (a) Imaginary part of $Y_{a}$. (b) $Q$ factor of $Y_{a}$.

(17), (21), (24), (28), (29), and (33) for extracting the equivalent multilayer resonant circuits for $Y_{p}$ and $Z_{g}$.

For the rational-approximation approach, it adopts the 3-dB bandwidth of $\left|Y_{a}\right|$ and $\left|Z_{b}\right|$ at the resonant frequencies identified in the direct-extraction procedure to estimate the complex starting poles to be used in the vector-fitting procedure. This action could avoid the ill-conditioning problems in vector fitting [25], [26], and consequently obtained the accurate poles,

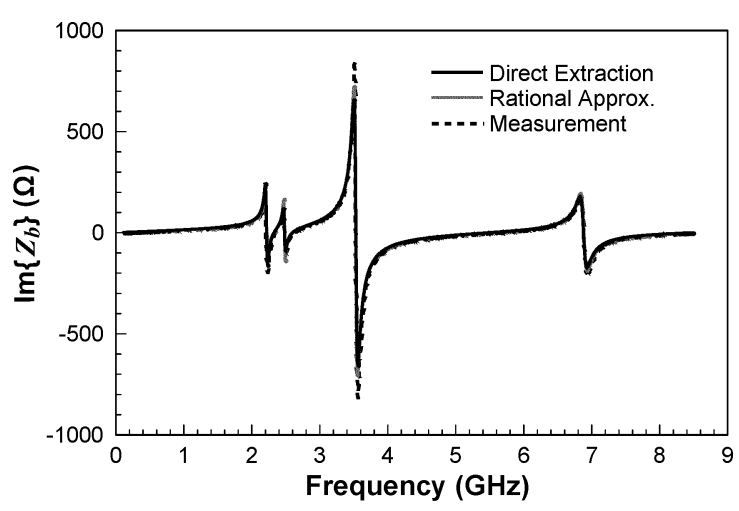

(a)

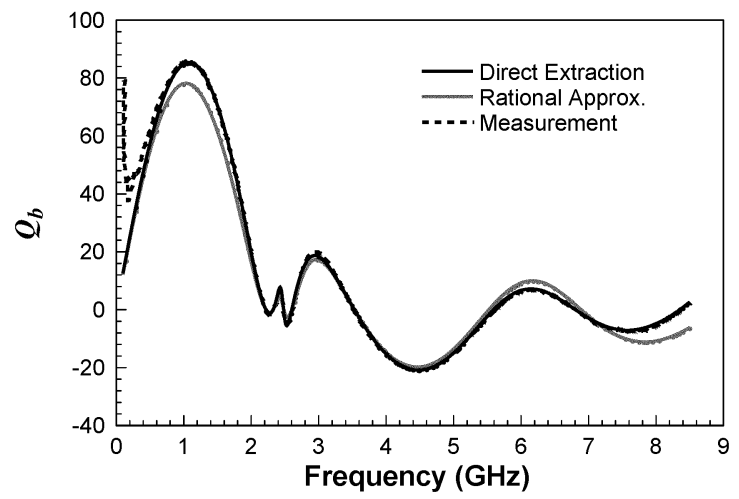

(b)

Fig. 11. Comparisons of the modeled results with measurements for the oneport network with input impedance of $Z_{b}$. (a) Imaginary part of $Z_{b}$. (b) $Q$ factor of $Z_{b}$.

residues, and coefficient constants listed in Table II very efficiently. Note that the real parts of all poles listed in Table II are negative, which can assure the stability of the fitting models for $Y_{a}$ and $Z_{b}$. Besides, for consideration of passivity in time-domain simulation, the positive real property of $Y_{a}$ and $Z_{b}$ has been also assured by checking all the residues and coefficient constants listed in Table II to satisfy the relation of positive semidefiniteness described in [28].

Since the modeled LTCC BPF has low-loss characteristics, the parameters $Y_{a}$ and $Z_{b}$ used in establishing the modified T-equivalent circuits clearly exhibit multiple resonances in the imaginary responses, as can be seen in Figs. 7 and 8. Identifying the resonant frequencies in the imaginary responses of $Y_{a}$ and $Z_{b}$ is greatly helpful to find good rational models with relatively low order. Therefore, there may be no such need to apply the advanced techniques like Cauchy's methods with an adaptive selection of sampling points and model order [13]-[15] or the model-order reduction methods [29], [30] for yielding the reduced-order rational models.

After substituting the parameters listed in Table II into (43)-(46), we found another set of the equivalent multilayer resonant circuits for $Y_{p}$ and $Z_{g}$. As for the equivalent series $L R$ circuits for $Z_{s 1}, Z_{s 2}$, and $Z_{z}$ formulated in (12)-(14) and (50)-(52) during the procedure of direct extraction and rational approximation, respectively, they were primarily extracted at low frequencies. 


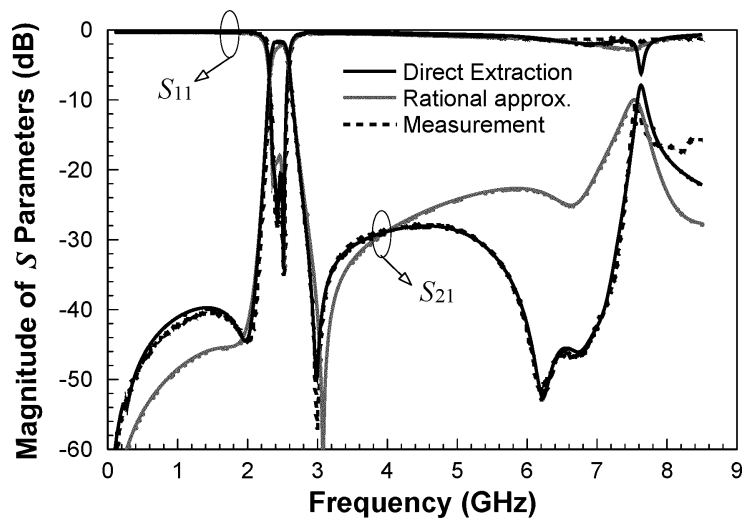

(a)

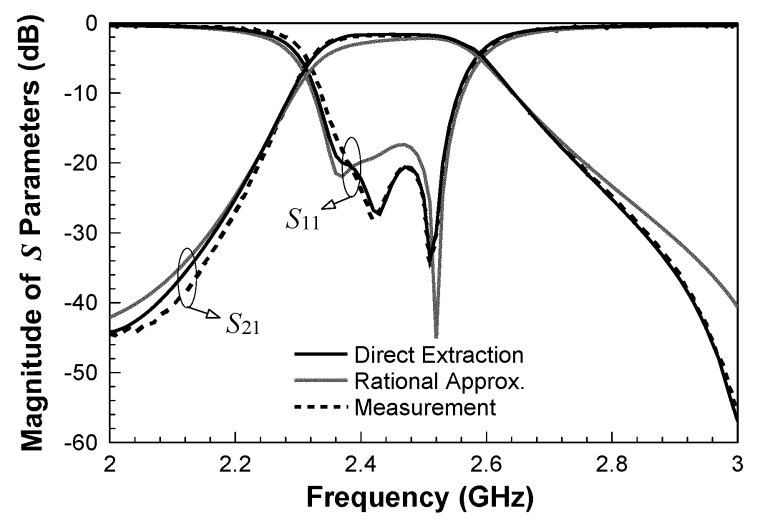

(b)

Fig. 12. Comparisons of the modeled results of $S$-parameter magnitudes with measurements for the 2.45-GHz LTCC BPF. (a) Magnitudes of $S_{11}$ and $S_{21}$ from 0.1 to $8.5 \mathrm{GHz}$. (b) Magnitudes of $S_{11}$ and $S_{21}$ from 2 to $3 \mathrm{GHz}$.

As a result, Fig. 9(a) and (b) shows the two-port modified T-equivalent circuit established based on direct extraction and rational approximation, respectively. Figs. 10 and 11 compare the modeling accuracy in the two decomposed one-port $\left(Y_{a}\right.$ and $Z_{b}$ ) networks between the two approaches. One can see in Figs. 10(a) and 11(a) that the modeled results of the imaginary responses of $Y_{a}$ and $Z_{b}$ from both approaches show excellent agreement with the measured results. However, a moderate discrepancy between the two approaches has been found in the modeled results of $Q_{a}$ and $Q_{b}$ shown in Figs. 10(b) and 11(b), respectively. Due to the specific use of the local maxima and minima points in resistance extraction, the direct-extraction approach can generate the modeled results for $Q_{a}$ and $Q_{b}$ having better agreement with measurements than the rational-approximation approach. This also implies that the modeled results for $Y_{a}$ and $Z_{b}$ using a rational approximation cannot satisfactorily account for the real responses.

Figs. 12-14 show comparisons of the modeled $S$-parameters and group delays with measurements between the two modified T-equivalent circuits shown in Fig. 9(a) and (b). It can be seen that the modeled results from direct extraction can achieve an impressive agreement with the measured results over the entire measurement frequency range up to $8.5 \mathrm{GHz}$. For the other modeled results using rational approximation with worse matching of the $Q_{a}$ and $Q_{b}$ parameters, a larger deviation from measurement has been found in the $S_{21}$-related responses, such as the

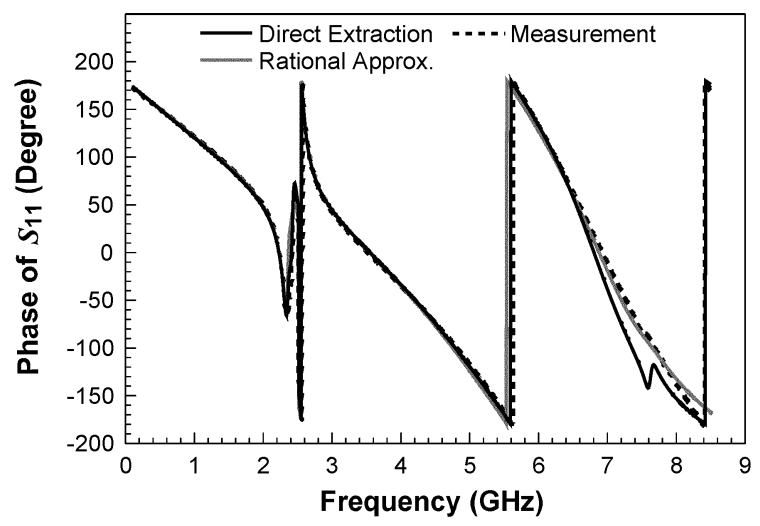

(a)

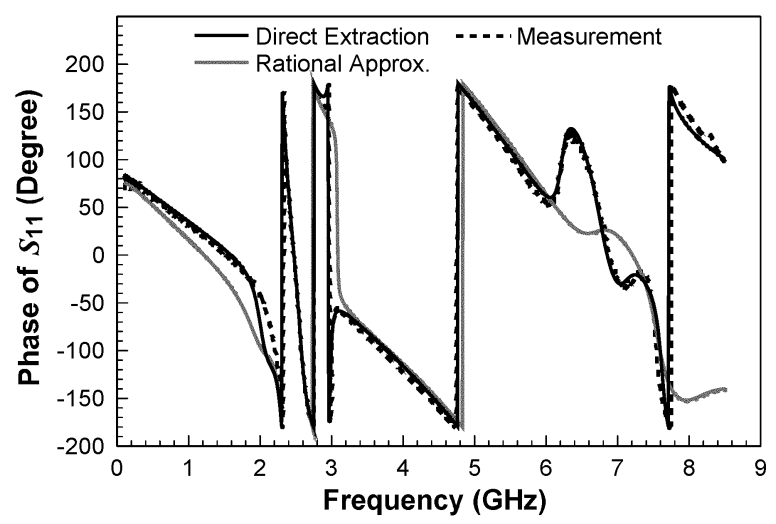

(b)

Fig. 13. Comparisons of the modeled results of $S$-parameter phases with the measurements for the 2.45-GHz LTCC BPF. (a) Phase of $S_{11}$ from 0.1 to $8.5 \mathrm{GHz}$. (b) Phase of $S_{21}$ from 0.1 to $8.5 \mathrm{GHz}$.

insertion losses in Fig. 12(a) and (b) and the phase and group delays in Figs. 13 and 14.

For general application of the proposed modified T-equivalent circuit topology to other types of filters, or even any microwave passive components in two-port configuration, here we attempt to outline a suggested model extraction procedure as follows.

Step 1) Find a low-frequency prototype with physical sense for the component to be modeled with the modified T-equivalent circuit.

Step 2) Convert the low-frequency prototype into an equivalent circuit in the modified $\mathrm{T}$ topology, as described in Section II.

Step 3) Expand the parallel- and series-feedback elements of the equivalent circuit in the modified $\mathrm{T}$ topology as multilayer resonators to account for the high-frequency resonance effects, as described in Section III.

Step 4) Decompose the two-port modified T-equivalent circuit into the two one-port circuits with input admittance of $Y_{a}$ for one one-port circuit and input impedance of $Z_{b}$ for the other. Both $Y_{a}$ and $Z_{b}$ data come from the measured or EM-simulated $S$-parameter data, as also described in Section III.

Step 5) Determine the circuit elements in $Y_{a}$ and $Z_{b}$ 's oneport circuits using the direction-extraction method 


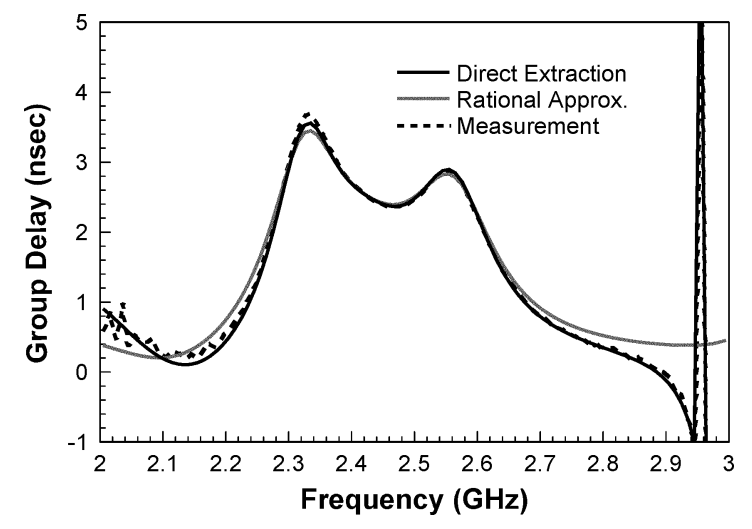

Fig. 14. Comparisons of the modeled results of group delay with the measurements from 2 to $3 \mathrm{GHz}$ for the 2.45-GHz LTCC BPF.

described in Section IV or the rational-approximation method described in Section V. To this step, all the circuit elements in the modified T-equivalent circuit are determined.

It is finally noted that the automatic generation of mathematically equivalent circuits for microwave passive components has been reported in the open literature [31]-[34] and even developed as commercial tools [35], [36]. In their typical procedure for modeling a two-port reciprocal component, rational approximation is applied to the three network parameters, i.e., $Z_{11}, Z_{12}\left(Z_{21}\right)$, and $Z_{22}$ or $Y_{11}, Y_{12}\left(Y_{21}\right)$, and $Y_{22}$ for establishing the equivalent circuits based on Cauer or Foster network synthesis techniques [37], [38]. When compared to the proposed modified T-equivalent circuits, the equivalent circuits established in their ways generally need more circuit elements to achieve a similarly large bandwidth. This is because the synthesis of modified T-equivalent circuits counts on modeling only two parameters, i.e., $Y_{a}$ and $Z_{b}$. Besides, their equivalent circuits consist of $R, G, L$, and $C$ elements often with many additional ideal transformers if generated using a Cauer structure [31], [32] or with negative-valued elements if generated using a Foster structure [33]-[36] and, thus, are not as comprehensible as the modified $\mathrm{T}$-equivalent circuits.

\section{CONCLUSION}

The proposed new equivalent circuit has been found suitable for broadband modeling of LTCC BPFs. This is because the equivalent circuit uses a modified $\mathrm{T}$ topology to well characterize the LTCC BPF prototype configurations. In addition, it includes the parallel- and series-feedback resonant circuits to appropriately account for the high-frequency resonance effects. These two resonant circuits can be expanded to meet any need for increased bandwidth. It is emphasized that this new equivalent circuit can be efficiently established because all the circuit elements can be determined from the measured $S$-parameters by means of either direct extraction or rational approximation. Consequently, in the example of modeling a $2.45-\mathrm{GHz}$ WLAN LTCC BPF, the modeled $S$-parameters have good agreement with the measured results over a frequency range more than three times larger than the BPF's passband center frequency.

\section{REFERENCES}

[1] K. M. Lakin, "Modeling of thin film resonators and filters," in IEEE MTT-S Int. Microw. Symp. Dig., 1992, pp. 149-152.

[2] S. Wang, W. G. Odendaal, and F. C. Lee, "Extraction of parasitic parameters of EMI filters using scattering parameters," in Proc. IEEE Ind. Appl. Soc. Conf., 2004, pp. 2672-2678.

[3] S.-G. Mao, M.-S. Wu, Y.-Z. Chueh, and C.-H. Chen, "Modeling of symmetric composite right/left-handed coplanar waveguides with applications to compact bandpass filters," IEEE Trans. Microw. Theory Tech., vol. 53, no. 11, pp. 3460-3466, Nov. 2005.

[4] K.-L. Wu, R. Zhang, M. Ehlert, and D.-G. Fang, "An explicit knowledge-embedded space mapping technique and its application to optimization of LTCC RF passive circuits," IEEE Trans. Compon. Packag. Technol., vol. 26, no. 2, pp. 399-406, Jun. 2003.

[5] M. A. Ismail, S. A. Panariello, Y. Wang, and M. Yu, "EM-based design of large-scale dielectric-resonator filters and multiplexers by space mapping," IEEE Trans. Microw. Theory Tech., vol. 52, no. 1, pp. 386-392, Jan. 2004.

[6] K.-L. Wu, Y.-J. Zhao, J. Wang, and M. K. K. Cheng, "An effective dynamic coarse model for optimization design of LTCC RF circuits with aggressive space mapping," IEEE Trans. Microw. Theory Tech., vol. 52, no. 1, pp. 393-402, Jan. 2004

[7] I. A. Eshrah, A. A. Kishk, A. B. Yakovlev, W. G. Glisson, and C. E. Smith, "Analysis of waveguide slot-based structures using wideband equivalent-circuit model," IEEE Trans. Microw. Theory Tech., vol. 52, no. 12, pp. 2691-2696, Dec. 2004.

[8] T. Thal, "Computer-aided filter alignment and diagnosis," IEEE Trans. Microw. Theory Tech., vol. MTT-26, no. 12, pp. 958-963, Dec. 1978.

[9] P. Harscher and R. Vahldieck, "Automated computer-controlled tuning of waveguide filters using adaptive network models," IEEE Trans. Microw. Theory Tech., vol. 49, no. 11, pp. 2125-2130, Nov. 2001.

[10] H.-T. Hsu, Z. Zhang, K. A. Zaki, and A. E. Atia, "Parameter extraction for symmetric coupled resonator filters," IEEE Trans. Microw. Theory Tech., vol. 50, no. 12, pp. 2971-2978, Dec. 2002.

[11] P. Harscher and R. Vahldieck, "Automated filter tuning using generalized low-loss prototype networks and gradient-based parameter extraction," IEEE Trans. Microw. Theory Tech., vol. 49, no. 12, pp. 2532-2538, Dec. 2001.

[12] M. Kahrizi, S. Safavi-Naeini, S. K. Chaudhuri, and R. Sabry, "Computer diagnosis and tuning of RF and microwave filters using modelbased parameter estimation," IEEE Trans. Circuits Syst., vol. 49, no. 9, pp. 1263-1270, Sep. 2002.

[13] A. Garcia-Lamperez, S. Llorente-Romano, M. Salazar-Palma, and T. K. Sarkar, "Efficient electromagnetic optimization of microwave filters and multiplexers using rational models," IEEE Trans. Microw. Theory Tech., vol. 52, no. 2, pp. 508-521, Feb. 2004.

[14] A. Lamecki, P. Kozakowski, and M. Mrozowski, "Efficient implementation of the Cauchy method for automated CAD-model construction," IEEE Microw. Guided Wave Lett., vol. 13, no. 7, pp. 268-270, Jul. 2003.

[15] S. F. Peik, R. R. Mansour, and Y. L. Chow, "Multidimensional Cauchy method and adaptive sampling for an accurate microwave circuit modeling," IEEE Trans. Microw. Theory Tech., vol. 47, no. 12, pp. 2364-2371, Dec. 1998.

[16] T.-S. Horng, J.-M. Wu, L.-Q. Yang, and S.-T. Fang, "A novel modified-T equivalent circuit for modeling LTCC embedded inductors with a large bandwidth," in IEEE MTT-S Int. Microw. Symp. Dig., 2003, pp. $1015-1018$.

[17] — - "A novel modified-T equivalent circuit for modeling LTCC embedded inductors with a large bandwidth," IEEE Trans. Microw. Theory Tech., vol. 51, no. 12, pp. 2327-2333, Dec. 2003.

[18] C.-T. Chiu, T.-S. Horng, H.-L. Ma, S.-M. Wu, and C.-P. Hung, "Super broadband lumped models for embedded passives," in Proc. 54th Electron. Compon. Technol. Conf., 2004, pp. 1104-1107.

[19] Y.-S. Tsai and T.-S. Horng, "Broadband single-stage models for microwave bandpass filters," in IEEE MTT-S Int. Microw. Symp. Dig., 2006, pp. 1771-1774.

[20] S. Kobayashi and K. Saito, "A miniaturized ceramic bandpass filter for cordless phone systems," in IEEE MTT-S Int. Microw. Symp. Dig., 1995, pp. 391-394.

[21] H.-S. Song and Y.-S. Lee, "A miniaturized $2.4 \mathrm{GHz}$ band multi-layer bandpass filter using capacitively loaded quarter-wavelength slow-wave resonator," in IEEE MTT-S Int. Microw. Symp. Dig., 2003, pp. 515-518. 
[22] C.-W. Tang, Y.-C. Lin, and C.-Y. Chang, "Realization of transmission zeros in combline filters using an auxiliary inductively coupled ground plane," IEEE Trans. Microw. Theory Tech., vol. 51, no. 10, pp. 2112-2118, Oct. 2003.

[23] C.-W. Tang, "Harmonic-suppression LTCC filter with the stepimpedance quarter-wavelength open stub," IEEE Trans. Microw. Theory Tech., vol. 52, no. 2, pp. 617-624, Feb. 2004.

[24] B. Lakshminarayanan, H. C. Gordon, and T. M. Weller, "A substratedependent CAD model for ceramic multilayer capacitors," IEEE Trans. Microw. Theory Tech., vol. 48, no. 10, pp. 1687-1963, Oct. 2000.

[25] B. Gustavsen and A. Semlyen, "Rational approximation of frequency domain responses by vector fitting," IEEE Trans. Power Del., vol. 14, no. 3, pp. 1052-1061, Jul. 1999.

[26] R. Neumayer, A. Stelzer, F. Haslinger, and R. Weigel, "On the synthesis of equivalent-circuit models for multiports characterized by frequency-dependent parameters," IEEE Trans. Microw. Theory Tech., vol. 50, no. 5, pp. 2789-2796, May 2002.

[27] G. Antonini, "SPICE equivalent circuits of frequency-domain responses," IEEE Trans. Electromagn. Compat., vol. 45, no. 3, pp. 502-512, Aug. 2003.

[28] R. Gao, Y. S. Mekonnen, W. T. Beyene, and S. A. Jose, "Black-box modeling of passive systems by rational function approximation," IEEE Trans. Adv. Packag., vol. 28, no. 2, pp. 209-215, May 2005.

[29] A. Odabasioglu, M. Celik, and L. T. Pileggi, "PRIMA: Passive reduced-order interconnect macromodeling algorithm," IEEE Trans. Comput.-Aided Des. Integr. Circuits Syst., vol. 17, no. 8, pp. 645-654, Aug. 1998.

[30] K. Krohne and R. Vahldieck, "On the application of model-order reduction in the fast and reliable optimization of microwave filters and diplexers," IEEE Trans. Microw. Theory Tech., vol. 52, no. 9, pp. 2285-2291, Sep. 2004.

[31] T. Mangold and P. Russer, "Full-wave modeling and automatic equivalent-circuit generation of millimeter-wave planar and multilayer structures," IEEE Trans. Microw. Theory Tech., vol. 47, no. 6, pp. 851-858, Jun. 1999.

[32] I. Timmins and K.-L. Wu, "An efficient systematic approach to model extraction for passive microwave circuits," IEEE Trans. Microw. Theory Tech., vol. 48, no. 9, pp. 1565-1573, Sep. 2000.

[33] M. Rencz, V. Székely, and A. Poppe, "A fast algorithm for the layout based electro-thermal simulation," in Proc. Des., Automat., Test in Eur. Conf. and Exhibition, 2003, pp. 1032-1037.

[34] T.-L. Wu, C.-C. Kuo, H.-C. Chang, and J.-S. Hsieh, "A novel systematic approach for equivalent model extraction of embedded high-speed interconnects in time domain," IEEE Trans. Electromagn. Compat., vol. 45, no. 8, pp. 493-501, Aug. 2003.
[35] “Signal Integrity Design Assistants (SIDEA) User's Guide,” Optimal Corporation, San Jose, CA, 2004.

[36] "Broadband SPICE product brochure," Sigrity Inc., Santa Clara, CA, 2004.

[37] F. L. Fontaine and S. Basu, "The partial realization problem for complex Hamburger series and complex lossless multiport networks," IEEE Trans. Circuits Syst., vol. 46, no. 1, pp. 161-177, Jan. 1999.

[38] R. Mohan, M. J. Choi, S. E. Mick, F. P. Hart, K. Chandrasekar, A. C. Cangellaris, P. D. Franzon, and M. B. Steer, "Causal reduced-order modeling of distributed structures in a transient circuit simulator," IEEE Trans. Microw. Theory Tech., vol. 51, no. 12, pp. 1179-1184, Dec. 2003.

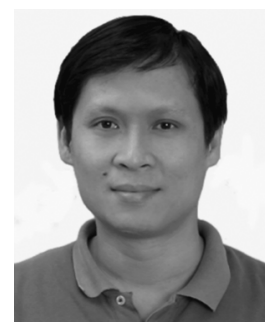

Yu-Shun Tsai was born in Kaohsiung, Taiwan, R.O.C. on March 3, 1963. He received the M.S.E.E. degree from National Chiao Tung University, Hsinchu, Taiwan, R.O.C., in 1991, and is currently working toward the Ph.D. degree in electrical engineering at National Sun Yat-Sen University, Kaohsiung, Taiwan, R.O.C.

His research interests include design and modeling of microwave components.

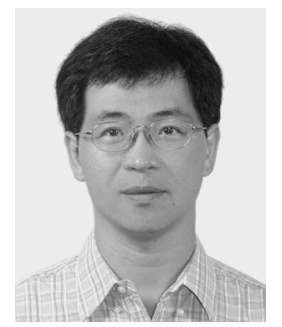

Tzyy-Sheng Horng (S'88-M'92-SM'05) was born in Taichung, Taiwan, R.O.C., on December 7, 1963. He received the B.S.E.E. degree from National Taiwan University, Taipei, Taiwan, R.O.C., in 1985, and the M.S.E.E. and Ph.D. degrees from the University of California at Los Angeles (UCLA), in 1990 and 1992, respectively.

He is currently a Professor with the Department of Electrical Engineering and also the Director of the Institute of Communications Engineering, National Sun Yat-Sen University, Kaohsiung, Taiwan, R.O.C. His research interests include RF and microwave integrated circuits, RF systems-on-package, and digitally assisted RF technology. 